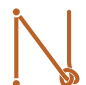

Volumen $6 \mathrm{~N} .{ }^{\circ} 45$ julio - diciembre de 2018 ISSN: 0122-4328

ISSN-E: 2619-6069 pp. $52-65$

\title{
Educación rural en Colombia: el país olvidado, antecedentes y perspectivas en el marco del posconflicto
}

\author{
Rural Education \\ in Colombia: the \\ Forgotten Country, \\ Background and \\ Perspectives in the \\ Post-conflict Context
}

\author{
Educação rural \\ na Colômbia: o \\ país esquecido, \\ antecedentes \\ e perspectivas \\ no contexto do \\ pós-conflito
}

\section{Andrea Parra Triana* Jairo Mateus Arbelaez** Zullybeth Mora Cubillos***}

Fecha de recepción: 20-08-18

Fecha de aprobación: 30-10-18

\section{PARA CITAR ESTE ARTÍCULO}

Parra, A., Mateus, J. y Mora, Z. (2018). Educación rural en Colombia: el país olvidado, antecedentes y perspectivas en el marco del posconflicto. Nodos y Nudos, 6(45), 52-65. doi:10.17227/nyn.vol6.num45-8320
Antropóloga Universidad de los Andes. Asesora. Fundación Empresarios por la Educación. andreaparratriana@gmail.com

Magister en Desarrollo Rural, Pontificia Universidad Javeriana. Docente tiempo completo ocasional. Universidad Pedagógica Nacional. jairomateusa@hotmail.com

***

Magister en Educación y Desarrollo Social, Centro Internacional para el Desarrollo Humano (Cinde) y Universidad Pedagógica Nacional. Docente, consultora e investigadora independiente. zullymoracubillos@gmail.com 


\section{RESUMEN}

En este documento se hace un recorrido sobre la implementación del Programa de Educación Rural Fase I y II del Ministerio de Educación Nacional, realizado con apoyo del Banco Mundial entre los años 2002 y 2015. Aqui se incluye una reflexión sobre aspectos pedagógicos, institucionales e investigativos, asociados con la apuesta de estos años de la educación rural para el pais, de modo que permite conocer algunos aciertos y oportunidades de mejoramiento de especial relevancia, en el marco de los esfuerzos nacionales y regionales por adelantar estrategias que apoyen las acciones derivadas de los acuerdos de paz.

Palabras clave: educación rural; políticas de educación rural en Colombia; Proyecto de Educación Rural

\section{ABSTRACT}

This document is intended to make a review on the implementation of the programme of Rural education phase I and II of the National Education Ministry, carried out with the support of the World Bank between 2002 and 2015. This review includes a reflection around teaching, research, and institutional aspects associated with the commitment of these years of rural education for the country, so that it allows to know some successes and improvement opportunities of special relevance in the context of the national and regional efforts to advance strategies that support the actions derived from the peace accords.

Key words: rural education; rural education policies in Colombia; Rural Education Project

\section{RESUMO}

Este documento tem como propósito fazer um percurso sobre a implementação do Programa de Educação Rural Fase I e II do Ministério de Educação Nacional, realizado com apoio do Banco Mundial entre os anos 2002 e 2015. Este percurso inclui uma reflexão ao redor de aspectos pedagógicos, institucionais e investigativos, sócios com a aposta destes anos da educação rural para o país, de maneira que permite conhecer alguns aciertos e oportunidades de melhoria de especial relevância, no marco dos esforços nacionais e regionais por adiantar estratégias que apoiem as acções derivadas dos acordos de paz.

Palavras-chave: educação rural; políticas de educação rural na Colômbia; Projeto de Educação Rural 


\section{Introducción}

La primera parte del documento presenta el contexto, desarrollo y productos del Programa de Educación Rural (PER), fase I, ejecutada entre 2002 y 2006, y posteriormente, la fase II, entre $2008^{1}$ y 2015 . También se hace un acercamiento a la respuesta que el Ministerio de Educación Nacional (men) le ha dado a la educación rural luego del cierre del PeR fase II, con énfasis en el Plan Especial de Educación Rural (PEer), construido como respuesta a los acuerdos de paz.

Por último, se presentan aciertos y oportunidades de mejoramiento, y se aportan elementos para la actual discusión alrededor de la educación rural en el país.

\section{Programa de Educación Rural fase I: Experiencias, saberes y producción de sentidos}

En el año 2000 el Ministerio de Educación Nacional (men) da inicio al Programa de Educación Rural (PER) que, en un proceso originalmente pactado a diez años, buscaba mejorar las condiciones de cobertura y calidad del servicio educativo en las zonas rurales del país, el fortalecimiento de la gestión y planificación de las entidades territoriales, y el mejoramiento de la capacidad del men para administrar y liderar la educación rural nacional (Perfetti, 2003).

En estos diez años se esperaba desarrollar tres fases de trabajo, cada una con un promedio de ejecución de tres años y medio, así: primera fase: implementación y aprendizaje; segunda fase: expansión y sostenibilidad, y tercera fase: expansión y consolidación. El ejercicio de temporalidad arrojó como valor de inversión US $\$ 120$ millones², los cuales serian asumidos de la siguiente forma: $50 \%$ por el Banco Mundial, $37 \%$ por el Gobierno nacional, y el $13 \%$ restante por las entidades territoriales participantes.

Este programa se adelantó como resultado de reflexiones previas; primero, la reacción al denominado Contrato Social Rural (que se da como respuesta a las marchas campesinas de 1996), que pretendía

1 En términos administrativos, la segunda fase comprendió los años 2008 a 2015. Sin embargo, la descripción presentada incluye información relacionada con el periodo 2010-2015, considerando que entre 2008 y 2009 se adelantaron acciones administrativas relacionadas con el acuerdo de préstamo con el Banco Mundial.

2 Solo se ejecutaron las dos fases iniciales. reorganizar las características y sentidos de la educación destinada a los sectores rurales del país. Y segundo, la elaboración por parte del MEN de una Consulta Nacional en 1998, que a partir de la realización de foros regionales y nacionales estimó la necesidad de concretar acciones particulares dirigidas a los territorios rurales, con el ánimo de mejorar y cualificar la prestación del servicio educativo, intentando disminuir las brechas de cobertura y calidad entre las zonas urbanas y rurales del país (MEN-BM, 2000). Finalmente, el documento del Consejo Nacional de Política Económica y Social (Conpes) 3056 del 10 de noviembre de 1999, que autoriza a la nación el desarrollo de un préstamo de US $\$ 20$ millones con el Banco Mundial para ejecutar el PER fase I.

Aunque este proceso previo incluyó jornadas de participación con diferentes sectores de la población nacional, la redacción del documento guía para la ejecución del programa no planteó una base conceptual sobre las concepciones de ruralidad, educación rural o desarrollo de las que partirían las acciones. Sin embargo, la lectura del Manual Operativo del Acuerdo de Préstamo (Men-BM, 2000) deja entrever en los postulados operativos para su implementación, los imaginarios de desarrollo del gobierno de turno que derivan en una lógica de ruralidad y educación centrada en el acceso a la escolaridad.

El PER definió para su accionar cuatro componentes de trabajo con los siguientes porcentajes de inversión: componente 1, relacionado con la ampliación de la cobertura y la calidad para la población rural entre los 5 y 17 años (62,5\%); componente 2, fortalecimiento de las capacidades de las entidades y alianzas (16,3\%); componente 3, mejoramiento de las condiciones de convivencia $(11,3 \%)$, y componente 4, mejoramiento de las condiciones para el desarrollo de la educación media (10\%).

Teniendo en cuenta que más de $60 \%$ de las acciones estaban asociadas al fortalecimiento de las sedes educativas a través del acceso al servicio y la calidad de la educación, a partir de la creación e implementación de distintos modelos educativos flexibles, como propuesta alternativa a la educación regular, se establecieron en el manual operativo tres niveles de gestión, con el propósito de concentrar las acciones 
en las sedes educativas como unidades estratégicas del proceso de implementación. Para el caso del nivel municipal se crearon las Unidades Operativas Municipales (UоM); a nivel departamental se establecieron las Alianzas Estratégicas Departamentales (AED); y, a nivel nacional se estableció la Unidad Coordinadora Nacional conformada por un equipo técnico integrado por profesionales del MEN.

Producto de la implementación de la fase I del PER durante el periodo 2002-2006), se destacan los siguientes resultados:

[...] aumentó la tasa de aprobación en 4.7 puntos porcentuales, disminuyó las tasas de reprobación en 1.4 puntos porcentuales y la deserción en 3.2 puntos al tiempo que mejoró los resultados en pruebas de calidad. Por último, cálculos de costo beneficio indican que el programa es altamente rentable con una tasa interna de retorno TIR cercana al $20 \%$. (Rodríguez, Sánchez y Armenta, 2007, p. 5).

[...] hacia finales del 2005, el PER se había ejecutado en 27 de los 32 departamentos colombianos. El programa había sido implementado en más de 6.000 sedes rurales, con 14.732 experiencias educativas que cobijaban a más de 400.000 alumnos. (Rodríguez, Sánchez y Armenta, 2007, p. 9).

Los equipos de trabajo en los niveles de gestión local, departamental y nacional, realizaron ejercicios diagnósticos que llevaron a la construcción e implementación de los modelos educativos flexibles para atender la problemática asociada a los componentes de acceso, deserción, calidad, convivencia y gestión municipal. Los modelos educativos flexibles, entendidos como:

[...] estrategias de cobertura, calidad, pertinencia y equidad del servicio público educativo, así como de permanencia de la población estudiantil en el servicio educativo, los cuales asumen los procesos de enseñanza aprendizaje dentro de la educación formal con alternativas escolarizadas y semiescolarizadas que se ajustan a las necesidades de los estudiantes en términos de tiempo, ubicación geográfica y condiciones de vulnerabilidad. Los modelos cuentan con apoyos adicionales al sistema regular, tales como la búsqueda activa de niños, niñas y jóvenes por fuera del sistema educativo, orientación psicosocial, alimentación escolar, kits escolares y canastas educativas conformadas con elementos pedagógicos y lúdicos, adaptados a los diferentes contextos, que buscan estimular al estudiante para que disfrute sus procesos de aprendizaje. Tienen explícitos los principios epistemológicos, psicológicos, pedagógicos y metodológicos que orientan su implementación, así como los procesos de gestión y administración, los cuales permiten su articulación con el proyecto educativo institucional - PEl, de los establecimientos educativos oficiales. (MEN y Silva Carreño Administración e Ingeniería 2014, p. 8).

Es importante mencionar que, si bien los modelos educativos flexibles permitieron la vinculación a la educación formal de un porcentaje importante de población que habitaba las zonas rurales, estos fueron asumidos como estrategias únicas para garantizar el derecho a la educación de los habitantes del campo. Aunque se solucionó un problema básico de acceso, se dejaron de lado otras posibilidades de la educación rural y en la mayoría de los casos la oferta se redujo al enfoque agrícola, coartando las posibilidades de expansión social y cultural de las comunidades y el país.

Como ya se mencionó, el espacio destinado para el fortalecimiento de las capacidades de los territorios y la descentralización administrativa se convirtió en un escenario de transferencia de los modelos educativos flexibles, restando fuerza al ejercicio de institucionalización y diálogo de las comunidades respecto a su territorio, a su educación y a las ruralidades. Lentamente se desdibujó la propuesta de educación rural como dispositivo para la transformación de las relaciones entre el centro y la periferia, la construcción de un desarrollo con enfoque territorial, y como un escenario privilegiado para la deconstrucción de discursos y prácticas asociadas a estereotipos del campo como espacio de pobreza y exclusión, debido a la ausencia de un modelo de desarrollo con enfoque humano:

[...] la satisfacción de las necesidades, la consecución de una calidad de vida y muerte dignas, el amar y ser amado, y el florecimiento saludable de todos, en paz y armonía con la naturaleza, para la prolongación de las culturas humanas y de la biodiversidad. El Buen Vivir o sumak kawsay supone tener tiempo libre para la contemplación y la emancipación, y que las libertades, oportunidades, capacidades y potencialidades reales de los individuos-colectivos se amplien y florezcan de modo que permitan lograr simultáneamente aquello que la sociedad, los territorios, las diversas identidades colectivas y cada uno -visto como un ser humano-colectivo, 
universal y particular a la vez- valora como objetivo de vida deseable (tanto material como subjetivamente, sin producir ningún tipo de dominación a un otro). (Ramírez, 2012, pp. 20-21).

Atendiendo a lo anterior, es importante destacar las acciones realizadas por algunas entidades territoriales, donde las autoridades locales de turno invirtieron recursos propios para el desarrollo del programa en las sedes educativas de su jurisdicción, y vincularon a otras entidades de carácter privado o mixto para financiar las propuestas del departamento, como referentes de autonomía local, capacidad de descentralización y compromiso respecto a la garantía del derecho a la educación.

Como se mencionó anteriormente, las acciones del programa llegaron a 27 de los 32 departamentos; se seleccionaron entidades territoriales y sedes educativas de acuerdo con unos criterios definidos en colectivo con el Banco Mundial. Para promover la intención de autonomía local, de acuerdo con el segundo objetivo del PER fase I, desde el nivel central se propuso a los integrantes de las Alianzas Educativas Departamentales (AED) evaluar la focalización de las sedes educativas a partir de los criterios definidos por el nivel central, situación que llevó a la vinculación y exclusión de algunas sedes educativas, replicando de nuevo la lógica del Estado ausente en muchos territorios.

Posteriormente, durante el MEN decide eliminar la figura de laS AED e institucionaliza la figura de convenios con los gobiernos territoriales, y concentrar las acciones en el desarrollo del primer objetivo: "aumentar la cobertura y calidad educativa en el sector rural para población entre los 5 y 17 años (desde preescolar hasta básica y secundaria)", con el propósito de expandir el programa a otros municipios y minimizar el impacto causado por la no atención de todas las sedes educativas. Concentrar las acciones durante la primera fase del programa permitió ampliar de manera exitosa la cobertura. Sin embargo, excluir temas como el tercer objetivo (mejorar las condiciones de convivencia en el sector, especialmente en la institución educativa), visibiliza el atraso del país respecto a los retos relacionados con la promoción de los derechos humanos, sexuales y reproductivos, la construcción colectiva del territorio desde las microrrealidades y la formación en participación y ciudadanía, subtemas que permiten la reconstrucción del tejido social en escenarios históricamente permeados por el conflicto armado y la ausencia del Estado.

En cuanto a las investigaciones producidas durante el PER fase I, no se encuentran estudios de carácter investigativo adicionales a la evaluación de impacto del programa adelantada por la Universidad de los Andes durante 2009. Los resultados más relevantes fueron presentados en la parte superior del artículo, relacionados con el crecimiento de la matrícula, la disminución de la tasa de deserción y repitencia. Adicionalmente, para efectos del presente artículo y análisis sobre la educación rural, se asume la producción de los modelos educativos flexibles como un espacio inicial de investigación sobre la educación rural en el pais. Durante la primera fase del PER, el MEN contrató personas jurídicas y naturales para construir las propuestas pedagógicas y didácticas asociadas a la noción alternativa de educación formal para la población de zonas rurales, con atención especial en la población de básica primaria y secundaria. De esta manera, el MEN asume los modelos educativos flexibles como bandera de la educación rural para el país.

A pesar de los esfuerzos por contextualizar los contenidos con las lógicas culturales de los territorios, el ejercicio se acercó tímidamente a las comunidades porque las estrategias se relacionaron de manera instrumental con el entorno, lo cual impidió en algunos casos generar dialéctica entre las propuestas de escuela impulsadas por los modelos educativos flexibles y las proyecciones de las comunidades. De nuevo, el campo y la ruralidad son asumidos como escenarios exclusivamente destinados a la producción agropecuaria y a la generación de recursos, y la educación a través de los modelos educativos flexibles, se ve como la posibilidad de materializar lo antes mencionado. La pertinencia para lo rural, entonces, se redujo a lo agropecuario dejando de lado otros accesos como a la ciencia o el arte, claves para generar equidad. La educación no fue pensada en el marco de una apuesta por el desarrollo territorial articulando a las comunidades y a los distintos sectores. 
Por otra parte, durante el proceso de implementación de la primera parte del PER, el docente es concebido como un agente vital para el desarrollo de las propuestas educativas, sirviendo de puente entre la escuela y la comunidad "el papel determinante que tiene la relación con la comunidad circundante en el reconocimiento y aceptación que pueda tener un maestro rural, y por ende, en el lugar de la escuela en la vida de una vereda o corregimiento" (Zamora, 2012, p. 20). Sin embargo, los avances respecto a la dignificación de la labor fueron mínimos, entre ellos: Decreto 3020 de 2002, "por el cual se establecen los criterios y procedimientos para organizar las plantas de personal docente y administrativo del servicio educativo estatal que prestan las entidades territoriales y se dictan otras disposiciones", donde se establecen diferencias entre la relación técnica de estudiantes para la zona urbana y la zona rural, y el Decreto 1171 de 2004, "por el cual se reglamenta el inciso 6 del artículo 24 de la Ley 715 de 2001 en lo relacionado con los estímulos para los docentes y directivos docentes de los establecimientos educativos estatales ubicados en áreas rurales de difícil acceso".

No obstante, dicha normatividad no generó las condiciones salariales esperadas, las oportunidades de formación necesarias para enfrentar los retos de los territorios rurales y el posicionamiento de la labor docente en las zonas rurales dentro de la lógica del sector educativo.

\section{Programa de Educación Rural fase II: tejido de saberes, vivencias y construcciones colectivas}

Durante la vigencia 2008, el MEN firmó la segunda fase de ejecución del PER, denominado formalmente "programa de fortalecimiento de la cobertura con calidad para el sector educativo rural, PER fase II", que culminó en diciembre de 2015 tras la aprobación de prórroga solicitada por esa cartera para dar cierre de acuerdo con los objetivos pactados entre las partes.

El PER fase II definió para su accionar tres componentes de trabajo en los cuales se incluyeron la totalidad de acciones del programa, estos fueron: a) mejoramiento de la capacidad de gestión de las secretarías de Educación municipales y departamentales; b) fortalecimiento de la gestión de las sedes educativas rurales para alcanzar resultados mejores y más equitativos en acceso, terminación de los niveles educativos y calidad del aprendizaje, y c). fortalecimiento de las capacidades del men para la atención del servicio educativo en las zonas rurales.

Con el primer componente, se esperaba construir y fortalecer capacidades en las Entidades Territoriales Certificadas (ETC) para promover servicios educativos equitativos, mecanismos de compensación para cerrar la brecha rural-urbana y la prestación del servicio educativo para las zonas más remotas y vulnerables del pais.

Para esto el PER fase II diseñó y extendió con las secretarías de Educación unos lineamientos para la formulación, gestión y monitoreo de planes de educación rural, intentando que desde las secretarias se fortaleciera una concepción de la ruralidad como territorio estratégico para el desarrollo y ejecución de la inversión pública. El instrumento pretendía también brindar herramientas a los territorios para hacer una lectura de contexto que permitiera cualificar la toma de decisiones por parte de las entidades territoriales respecto las nociones, prácticas y retos sobre la relación entre ruralidad y educación. Los lineamientos estaban organizados en cuatro partes: la prime$\mathrm{ra}$, concepciones y contexto sobre la ruralidad y las políticas educativas; la segunda, estrategias para la formulación de los planes; la tercera, la importancia del seguimiento durante la ejecución, y la última, relacionada con la evaluación de la implementación.

Con el segundo componente, se buscaba fortalecer las capacidades pedagógicas de docentes, directivos docentes e instituciones formadoras de docentes: Escuelas Normales Superiores (ENS), para desarrollar una mejor comprensión de las necesidades educativas de las zonas rurales, debido a que un número significativo de docentes que se desempeñan en el campo, provienen de estas instituciones. El ejercicio se concentró en profundizar en el rendimiento escolar y los resultados de aprendizaje de los estudiantes, a través de ejercicios de acompañamiento situado a docentes y directivos, cualificación de los Modelos 
Educativos Flexibles (meF) y el reconocimiento de las ENS como formadoras de formadores en el país.

Es importante resaltar que, dentro de este componente, el programa contó con el desarrollo de una línea de trabajo asociada a la promoción de la educación propia de los pueblos indígenas y afrodescendientes, a partir del apoyo para la construcción de modelos pedagógicos por parte de las comunidades.

Finalmente, el tercer componente desarrolló investigaciones que ayudaban al MEN a la comprensión del sector rural, en temas como la gestión educativa en territorios, las alternativas para la prestación del servicio educativo en zonas de población dispersa, el estudio para la formulación de un plan de incentivos para docentes, y la cualificación de los $\mathrm{MEF}^{3}$.

A partir del desarrollo de estos componentes se deja entrever una concepción del territorio rural y de la ruralidad, que en términos prácticos fue estimado según como este era entendido por el Departamento Administrativo Nacional de Estadística (DANE) para la época, estableciendo que las áreas urbanas correspondian a las cabeceras municipales y las áreas rurales a aquellas no incluidas dentro del perímetro de la cabecera municipal (Rojas, 2011). La concepción de lo rural para la toma de decisiones fue la de cabecera-resto, lo que implicó, por ejemplo, que las acciones del PER se focalizaran exclusivamente en las sedes educativas no localizadas en las cabeceras urbanas o municipales, en las que se brinda principalmente la básica primaria.

Este escenario llevó a una concepción fragmentaria, no solo de la ruralidad sino del sistema educativo, hecho que dificultó la inversión económica para adelantar procesos de formación o el mejoramiento de materiales y recursos educativos, de sedes localizadas en las cabeceras municipales pero que en últimas, atendían, especialmente en el nivel de educación básica secundaria y media, a pobladores de las zonas rurales.

Por otro lado, teniendo en cuenta los altos costos en la atención de las sedes rurales, el porcentaje de

3 En total durante la fase II del PER se hicieron nueve estudios, concernientes a: Relación Estudios Realizados Mejoramiento, Estudio Uso Tiempo Aula, Estudio Evaluación Materiales, Estudio Modelos Educativos Flexibles, Estudio Fusión Sedes Rurales 2013, Estudio Evaluación Textos Matemáticas. Estudios Evaluación Textos Lenguaje, Estudio Jornada Única, Estudio Materiales Guias. sedes focalizadas, durante la segunda fase del proyecto, llega apenas al $22 \%$ sobre el total de sedes (7717 sedes, ubicadas en 78 municipios de 36 ETC). El impacto de las acciones del PER en cada uno de los departamentos focalizados es diversa; una vez más, la lógica de la focalización impide que se garantice el derecho a la educación de todos los habitantes.

De otra parte, el PER buscaba cualificar la planificación y gestión educativa en las entidades territoriales. Para este propósito el MEN y Corpoeducación (2012) asumen

El desarrollo rural con enfoque territorial, que parte de la premisa de que es necesaria la participación activa de los actores locales del territorio para garantizar un proceso endógeno de cambio con el apoyo de los niveles público y privado tanto nacionales como, dado el caso, internacionales. (MEN y Corpoeducación, 2012, p. 10).

El MEN asumió un papel de puente entre las necesidades locales autodiagnosticadas, las acciones deseadas desde las regiones y los fondos presupuestales nacionales derivados del acuerdo de préstamo. Sin embargo, la concepción de ruralidad ya mencionada llevó en la práctica a que, aunque las entidades territoriales planearan de manera autónoma y participativa sus perspectivas de desarrollo educativo rural, tuvieran que hacerlo desde la visión desarticulada y fragmentada de la que el MEN partía, dificultando la comprensión e integración de los flujos y dinámicas sociales, económicas y culturales de las cabeceras municipales, las capitales departamentales y las poblaciones dispersas, convirtiendo el programa en un listado de acciones puntuales que difícilmente podía derivar en un ejercicio de mejoramiento integral. Puede afirmarse entonces que el PER se desarrolló a partir de la oferta de modelos y proyectos específicos pensados desde el nivel central, y ejecutados en los territorios generalmente por operadores privados. Esto cuestiona el desarrollo de capacidades locales que dejó el proyecto en las entidades territoriales, porque generalmente las acciones derivadas del préstamo estuvieron a cargo de un solo profesional, ubicado en un área específica de la Secretaría de Educación, lo cual generó una manera de entender y asumir la educación rural en las ETC, a pesar de los esfuerzos de generar lazos de trabajo y articulación interinstitucional, 
a través de espacios como: los comités de educación rural. Podría decirse, más bien, que las capacidades quedaron en los operadores encargados de ejecutar los modelos y proyectos en los territorios.

Es importante mencionar que durante las dos fases del PER, el MEN estableció convenios de cooperación interadministrativa con 36 de las 97 etc con que cuenta el país. El criterio para la focalización de estas ETC obedeció a su interés en participar del programa, luego de un proceso de divulgación y promoción de este con las 97 ETc. Esto conllevó a que algunos departamentos con alta presencia de ruralidad y ruralidad dispersa no accedieran al establecimiento de convenios por falta de voluntad política de los gobiernos territoriales de turno.

Estos convenios permitieron establecer compromisos de ambas partes; el MEN, bajo la figura de fiducia, conformó un fondo común donde reposaron los recursos asignados por el programa a cada ETC, destinó un equipo para acompañar la gestión y seguimiento del programa, así como para adelantar los procesos de contratación con terceros para las acciones derivadas de los planes de educación rural de cada ETc. De otra parte, las ETc se comprometieron, entre otros, a: conformar un equipo de educación rural como interlocutor con el desarrollo del programa y destinar una contrapartida económica desde recursos propios, que en la mayoría de los casos estuvo entre el $20 \%$ y $30 \%$ del valor total del convenio. La iniciativa de conformación de un equipo de educación rural terminó, casi siempre, reduciéndose a la presencia de un encargado del tema, dificultando su institucionalización a nivel local. Esta dinámica se produjo debido al volumen de responsabilidades de las entidades versus el número de profesionales asignados para su implementación y evaluación. Adicionalmente, en la gran mayoría de los casos, primó la ausencia de la educación rural como una categoría y acción importante para la gestión educativa, y como un escenario estratégico para el desarrollo local y regional. Generalmente, la educación rural deambuló entre los lugares comunes de acceso y calidad educativa, y no logró mayor desarrollo conceptual y metodológico respecto a las lógicas propias de los territorios rurales, como se ha expresado a lo largo del documento.

Por tanto, los beneficiarios directos del programa fueron los docentes, directivos, estudiantes y funcionarios de las secretarías de Educación de las 36 ETC mencionadas. De forma indirecta, las 97 ETC beneficiarias de las nuevas lógicas de formación docente, como el Desarrollo Profesional Situado, la cualificación de algunos modelos educativos flexibles y herramientas metodológicas como el documento Manual para la formulación de planes y ejecución de planes de educación rural (MEN y Corpoeducación, 2012), que ofrece lineamientos para la formulación y gestión educativa rural.

\section{La apuesta del MEN, entre el acuerdo de paz y los retos del país rural}

Una vez finalizado el acuerdo de crédito en 2015, un grupo alojado en el área de cobertura se encarga de asumir los procesos relacionados con la educación rural en el país.

Los acuerdos de paz de La Habana y la Misión Rural habian puesto sobre la mesa la urgencia de voltear la mirada hacia estos territorios, históricamente abandonados y víctimas del conflicto armado. Los acuerdos, en el marco de la reforma rural integral, incluyeron el compromiso de elaborar para los territorios priorizados para el posconflicto, unos planes especiales en todos los temas críticos para estas regiones, incluida la educación. El MEN asume la tarea de empezar a consolidar lo que denominó el Plan Especial de Educación Rural (PeER), que se plantea desde el reconocimiento de las brechas entre lo urbano y lo rural, y la necesidad de promover estrategias pertinentes para resolver los problemas de permanencia, calidad, acceso y cobertura de los habitantes del campo. En cuanto a las estrategias pertinentes, estas se entinden como un conjunto de herramientas que buscaban garantizar el derecho a la educación de la población de las zonas rurales, atendiendo a las dinámicas y lógicas de los distintos territorios.

La propuesta nace en el marco de una apuesta por la equidad y la justicia social en el país. El MEN abre la discusión sobre los ejes del plan con organizaciones 
de la sociedad civil, donde se reconocen los esfuerzos hechos hasta el momento, a través de la implementación del PER fases I y II, y estrategias como el Programa Todos a Aprender (PTA), ejecutado en algunas de las zonas rurales. Sin embargo, tras el balance de la situación, a partir de la caracterización de las brechas en cada uno de los niveles de escolaridad, quedan en evidencia las cifras dramáticas y lo lejos que está el país a la hora de garantizar el derecho a la educación de sus habitantes rurales, el MEN propone una serie de lineamientos estratégicos para atender a estas poblaciones, con foco en aquellas más afectadas por el conflicto armado.

Las estrategias propuestas desde el plan se construyen a partir de un ejercicio previo elaborado por el MEN denominado "colegio 10". Se trata de una estrategia que presenta las características que desde el Ministerio se le atribuyen a un colegio ideal, en términos de cobertura, permanencia y calidad educativa en la lógica del sistema escolar regular. Bajo las mismas dimensiones formuladas para el "colegio 10 urbano", se propone entonces la adaptación para el "colegio 10 rural". Las dimensiones que sirven de base para la elaboración de las propuestas son: ambiente escolar y bienestar, infraestructura y dotación, pedagógico y académico, docentes y directivos docentes, y administrativa. En el centro del proceso se sitúa el estudiante.

La última versión del documento entregada en 2018 recoge los aportes de las diferentes organizaciones no gubernamentales convocadas para su lectura, y da un paso importante en la construcción de la apuesta por una mirada de la educación particular para lo rural. Aparecen en la propuesta temas como la contextualización de la normatividad de transporte y alimentación escolar a las características de las zonas rurales, la necesidad de generar procesos de titularidad de tierras, la apertura de espacios comunitarios de arte en la escuela o el rediseño del concurso de méritos e incentivos para maestros rurales. Elementos que han aparecido como necesarios en varias propuestas de política elaboradas desde la sociedad civil.

La propuesta, sin embargo, deja por fuera asuntos estructurales necesarios para garantizar el derecho de la educación de la población rural relacionados, por ejemplo, con la autonomía, generación de capacidades y toma de decisiones territoriales. Una vez más, como sucedió en el diseño de las dos fases del PER, no se evidencia una apuesta que desde lo conceptual y metodológico sitúe la manera en que, desde el Ministerio de Educación, se concibe la ruralidad y la educación rural. De la misma manera que sucedió con el PER, se proponen estrategias puntuales que no están articuladas con una apuesta de política pública o de institucionalización de la educación rural en el MEN y en las secretarías de Educación. El plan da una respuesta coyuntural, situada en los municipios priorizados para el postconflicto, pero el país sigue sin contar con una política pública educativa que responda a las diversidades de los territorios rurales.

Se mencionó anteriormente que la propuesta de calidad para la educación rural había concentrado sus mayores esfuerzos en las dos etapas del PER, en el diseño e implementación de modelos educativos flexibles, y se planteó la necesidad de ampliar este espectro; incluso de hacerse preguntas sobre las caracteristicas de la flexibilidad que requieren estos territorios, en especial las zonas más dispersas. Esta propuesta también lo hace; en gran medida, la solución brindada para garantizar el derecho a una educación de calidad se centra en los modelos y en la creación de instrumentos (guías, cartillas, elaboración de orientaciones, reglamentación de escuelas de familias, kits de materiales educativos), que, si bien son de gran importancia, no resuelven solos la inmensa brecha en términos de calidad.

La calidad educativa no puede ser considerada una categoría exclusiva de la escolaridad, debe pasar por el cuerpo de los actores que habitan el territorio, permear la cotidianidad de las comunidades y poner el diálogo las lógicas de vida de la población rural con otras lógicas de desarrollo, lo cual permite ampliar el horizonte de sentido respecto al mundo de la vida de las comunidades, buscar elementos para armonizar las relaciones dentro y fuera de sus territorios y construir las estrategias para mejorar la calidad de vida del país. En este sentido, la pedagogía se convierte en un elemento fundamental para reconfigurar la categoría de calidad educativa y las relaciones que se tejen en las zonas rurales, donde los aprendizajes circulan en el territorio, los agentes educativos son diversos y la educación no es un tema exclusivo de la 
escuela, si bien esta es un lugar estratégico, no es el único donde se movilizan los aprendizajes.

Otros aspectos como la educación media, la conformación de comunidades de aprendizaje de docentes o el papel de los directivos docentes no se desarrollan con la profundidad suficiente para entender cómo se llevarán a cabo, sobre todo en la ruralidad dispersa del país, históricamente excluidas de la vivencia de los derechos y generalmente, azotadas por el conflicto y las múltiples violencias. En el caso del PEER, al igual que en las dos fases del PER, el territorio, sus apuestas de desarrollo rural y el lugar de la educación como motor para garantizar este desarrollo parecen diluirse en un listado de estrategias que no se concretan todavía en una política pública para la ruralidad, tan necesaria para el país.

\section{Aciertos y oportunidades}

La implementación del PER significó para el país un esfuerzo por pensar y construir nuevas formas de relación con los territorios rurales. En ese camino recorrido se encuentran algunos aprendizajes importantes para este momento histórico del país. A continuación, se presentan las lecciones aprendidas durante el proceso, desde la perspectiva de los autores de este escrito, en calidad de profesionales de la educación rural. Los aportes se plantean en términos de aciertos que valen la pena considerar para dar continuidad dentro del ejercicio de política pública rural para el pais. Posteriormente, se exponen las oportunidades de mejoramiento, con el propósito de evitar la repetición en futuras formulaciones e implementaciones de la política, como el caso del plan de especial de educación rural.

\section{Aciertos}

» La apuesta del MEN para formular y ejecutar un modelo operativo para la atención de la población rural puso en la agenda del país la situación relacionada a la prestación del servicio educativo en las zonas rurales.

»El diseño del Per planteó por lo menos el reconocimiento de generar alternativas educativas diferenciadas para la ruralidad del país en el ámbito central y en las secretarias de Educación que estuvieron vinculadas con el proyecto, a través de los siguientes procesos: cualificación a los docentes y directivos docentes in situ durante la segunda fase del PER; reconocimiento y acompañamiento a algunas comunidades indigenas y afro en la construcción e implementación de los planes de educación propia asociados a sus planes de vida, y producción pedagógica alrededor de los modelos educativos flexibles en el marco de la primera pase del PER, y su revisión durante la segunda fase. tuado para el mejoramiento de la profesionalización de los docentes y directivos. Frente a este logro, vale la pena generar análisis que conlleven a evaluar cuál fue la incidencia de estos estudios en la toma de decisiones del MEN y las secretarías de Educación en cuanto a la educación rural.

» De otra parte, es importante valorar los esfuerzos realizados durante la primera fase, centrada en el acceso, debido a la baja vinculación de la población de las zonas rurales en el sistema educativo; y la segunda fase, en la apertura al componente de calidad educativa

\section{Oportunidades de mejoramiento}

» La educación rural se limitó a la implementación de modelos flexibles, con lo cual se desconoció la posibilidad de integrar las apuestas locales y se planteó un rol docente muchas veces reducido al trabajo en el aula.

" Sin desconocer el avance que permitieron los modelos educativos flexibles en términos de acceso en el pais, y los buenos resultados en el caso del Modelo Escuela Nueva en algunas regiones, desde el PER no se lograron legitimar otras alternativas de escolaridad que respondieran mejor a las dinámicas territoriales. Se podria haber dado acompañamiento a alternativas que ya se venía desarrollando en los territorios. En este sentido, vale la pena ampliar las posibilidades que, desde 
el Estado, se ha dado a la sistematización de experiencias como "una metodología que permite la visibilización de lo que hegemónicamente ha permanecido ausente y de esta manera busca resignificarlo y legitimarlo, potenciando de este modo la producción de saberes desde la experiencia de las comunidades" (Rodríguez, 2018, p. 17).

Las propuestas generadas para la vinculación de las entidades territoriales no certificadas no permitieron su participación directiva y activa, debido al modelo de gestión propuesto para la implementación y las apuestas del programa centradas en el acceso y permanencia de la población escolar, con lo que se perdió de vista otras dimensiones de la educación rural. Se trató de una propuesta, que, aunque en su concepción contó con la mirada territorial, estuvo ejecutada desde el centro del pais, con enormes dificultades de tipo administrativo que pasan por el escaso conocimiento real de los territorios.

» El Estado no cuenta con un modelo de gestión que permita dar respuesta oportuna y coordinada a las necesidades de la población en zonas rurales, de manera que los tiempos de ejecución coincidan con los cronogramas de planeación realizados desde las secretarías de Educación. Lo anterior obedece a la ausencia de una política pública de educación rural que sobrepase las lógicas y formas de accionar de los gobiernos de turno, propias de las dinámicas de la gestión pública del país. Esta situación, por ejemplo, derivó en que la implementación de algunos de los proyectos del programa de educación rural no coincidía con los calendarios escolares, o su ejecución se dio muchos meses después de lo presupuestado, cuando las condiciones de las sedes educativas se habían modificado, por ejemplo, con cambios dentro sus equipos docentes.

» El PER no incluyo desde el inicio de sus actividades una línea estratégica relacionada con la expansión e institucionalización de la educación rural, a través de la vinculación de actores, organizaciones e instituciones competentes, ni tampoco una estrategia de divulgación de las acciones desarrolladas. Por ejemplo, los estudios e investigaciones realizados no lograron integrarse a las dinámicas y procesos de investigación de instituciones de educación superior, organizaciones civiles y grupos de investigación.

» Las intervenciones en el marco del PeR no tuvieron una dimensión nacional, a pesar de su amplia cobertura. tampoco se logró construir un marco de política educativo, más allá de las acciones concretas llevadas a cabo en los territorios, como la implementación de modelos flexibles. Como resultado, la evaluación de resultados de la segunda fase del PER plantea que "los factores que limitan la atención adecuada de la educación rural empiezan por la ausencia de una política, marco normativo y presupuesto específico para la educación rural" (MEN y Unión Temporal Econometría - SEl, 2015, p 18). Se recomienda en esta evaluación, además, la creación de un documento Conpes para la educación rural. Solo hasta el cierre de la segunda fase se plantearon algunos lineamientos de política pública para la educación rural.

» El per no se cuestionó ni hizo apuestas conceptuales alrededor de asuntos clave para la ruralidad, empezando por la concepción misma de la educación rural. En esta mirada, vale la pena cuestionarse por la flexibilidad requerida para atender las necesidades de la población rural. Es una flexibilidad que no se agota en la implementación de los modelos, sino que desde lo conceptual y metodológico se debe establecer un diálogo cercano con las necesidades reales de los territorios.

» Si bien se avanzó en la elaboración de propuestas educativas propias de las comunidades indígenas y afro, faltó acompañamiento y continuidad a estas propuestas, así como la mirada sobre el lugar que ocupan los grupos étnicos en el marco de una apuesta por la educación rural del país.

La política y el trabajo con internados estuvo ausente de esta mirada hacia la educación rural del país. A pesar de ser una respuesta para el acceso al servicio educativo de población rural dispersa, la propuesta no cuenta con lineamientos de política ni condiciones que garanticen la dignidad, 
la seguridad integral de los niños, niñas y adolescentes, ni el desarrollo comunitario. Por el contrario, vulnera sus derechos fundamentales, y la posibilidad de establecer espacios que fortalezcan los lazos comunitarios y el territorio.

» Los esfuerzos respecto a la educación rural en el pais han dependido de créditos externos. No se ha adoptado en el men una instancia permanente que se encargue de la educación para la gente del campo con una mirada distinta en cuanto a la calidad educativa, las potencialidades del territorio y la gestión educativa centrada en el desarrollo de las capacidades de los distintos actores. Según la evaluación de resultados de la implementación de la segunda fase del PER, más de la mitad de las entidades territoriales participantes reconocen que a pesar de que se fortalecieron los responsables de la educación rural en las secretarías de Educación, esta capacidad quedó en cabeza de una sola persona y no de la entidad.

» La educación rural del país requiere de respuestas contundentes, no es posible seguir abordando los problemas con las mismas soluciones, es importante redefinir y reconfigurar las relaciones y las lógicas de Colombia como un país eminentemente rural.

\section{Referencias}

Ministerio de Educación Nacional (MEN) y Banco Mundial (BM) (2000). Manual Operativo del Acuerdo de Préstamo Crédito No. 7540-Co. Documento de trabajo. Bogotá.

Ministerio de Educación Nacional y Corporación para el Desarrollo de la Educación (Corpoeducación) (2012). Manual para la formulación de planes y ejecución de planes de educación rural: calidad y equidad para la población de la zona rural. Bogotá.

Ministerio de Educación Nacional (men) y Silva Carreño Administración e Ingeniería Ltda. Sca. (2014). Lineamientos técnicos, administrativos, pedagógicos y operativos del proceso de implementación de los modelos educativos flexibles del Ministerio de Educación Nacional. Bogotá.
Ministerio de Educación Nacional y Unión Temporal Econometría - Sistemas Especializados de Información (sel) (2015). Evaluación de los resultados de la implementación del programa de fortalecimiento de la cobertura con calidad para el sector educativo PER fase II, en su población beneficiaria identificando los efectos (esperados y no esperado) de la intervención sobre sus variables objetivo. Bogotá.

Perfetti, M. (2003). Estudio sobre la educación para la población rural en Colombia. En Food and Agriculture Organization, Educación para la población Rural en Brasil, Chile, Colombia, Honduras, México, Paraguay y Perú (pp. 164-216). Viale delle Terme di Caracalla: FAO.

Rodríguez, E. (2018). La sistematización de experiencias educativas en la pedagogía decolonial. Nodos y Nudos, 5(44), 15-34.

Rodríguez, C., Sánchez, F. y Armenta, A. (2007). Hacia una mejor educación rural: impacto de un programa de intervención a las escuelas en Colombia. Documento CEDE. Bogotá: Universidad de los Andes.

Rojas, J. (2011). La ruralidad en Colombia: una aproximación a su cuantificación. Revista IB, 1(1). Bogotá: DANE.

Ramirez, A. (2012). 'Cuidadoras in-visibles' Mujeres latinoamericanas trabajadoras del cuidado en Vitoria-Gasteiz, otra expresión de la crisis del Estado del Bienestar. En 25 años del Forum de Política Feminista y de Feminismo 1987-2012 (pp. 135-144). Forum Feminista María de Maetzu.

Zamora, L. (2012). Abordajes en la formación de educadores para el ejercicio de la docencia rural. Bogotá: Editorial Códice. 


\section{Diálogo del conocimiento}

Los autores en este artículo aportan elementos para reflexionar sobre los retos que tiene el pais frente a los acuerdos de paz y, en particular, a la creación de un Plan Especial de Educación Rural (PEER). En el escrito se logra una sintesis puntual sobre los avances y desaciertos que dejó la implementación del Programa de Educación Rural (PER), el cual buscó mejorar el servicio educativo que se presta en zonas rurales de Colombia. Andrea Parra, Jairo Mateus y Zullybeth Mora enfatizan en la necesidad de reconocer, en el marco de una política pública educativa para el sector rural, las dinámicas sociales existentes en las diferentes regiones rurales del pais y los riesgos que se corren al construir dicha política de manera centralizada, que se fija exclusivamente en parámetros de cobertura y de gestión administrativa.

Es necesario continuar el análisis de experiencias educativas desarrolladas con comunidades rurales. Es claro que trabajos como este aportan insumos para no caer en los errores del pasado y construir una politica educativa integral enfocada al sector rural que aporte al bienestar de las comunidades, al territorio y a los procesos culturales que alli están presentes. Este tipo de reflexiones son la voz que nos recuerda que el pais no puede dejar pasar por alto la oportunidad de construir una política educativa rural que ayude a saldar deudas históricas que tenemos con las comunidades rurales del país.

Camilo Valderrama Universidad Pedagógica Nacional cvalderrama@pedagogica.edu.co 